Article

\title{
Stability analysis of a single-species Markovian jumping ecosystem
}

\author{
Ruofeng Rao ${ }^{1,2}$ \\ 1 Department of Mathematics, Chengdu Normal University, Chengdu 61130, China; ruofengrao@163.com or \\ ruofengrao@cdnu.edu.cn \\ 2 Institute of Financial Mathematics, Chengdu Normal University, Chengdu 61130, China \\ Received: 18 August 2021
}

\begin{abstract}
In this paper, impulsive control on a single-species Markovian jumping ecosystem leads to a stability criterion, and the newly-obtained theorems improve the related existing results. Numerical examples illuminate the effectiveness.
\end{abstract}

Keywords: a single-species ecosystem; variational methods; global stability; reaction-diffusion ; Sobolev spaces

\section{Introduction}

As pointed out in [1], the following Logistic system has been widely concerned and studied due to its importance in the development of ecology:

$$
\frac{d \mathfrak{Z}}{d t}=\mathfrak{R} \mathfrak{Z}(t)\left(1-\frac{\mathfrak{Z}(t)}{\mathfrak{K}}\right),
$$

where $\mathfrak{Z}(t)$ represents the density or quantity of population $\mathfrak{Z}$ at time $t, \mathfrak{R}>0$ and $\mathfrak{K}$ represent the intrinsic growth rate of population and environmental capacity, respectively. Because all solutions of nonlinear ecosystem are difficult to be given accurately, people pay more attention to the long-term dynamic trend of ecosystem, i.e., the long-term trend of population density (see, e.g. [1-5]). People especially want to know whether the population will tend to a positive constant after a long time, which is related to the final long-term existence of the population. For example, the authors of [2] investigated the long time behavior of the following stochastic ecosystem for a single-species:

$$
d \mathfrak{Z}=\mathfrak{Z}[a-b \mathfrak{Z}] d t+\gamma \mathfrak{Z} d B(t) .
$$

Animal populations will inevitably spread because of climate, foraging and random walking. And hence the reaction-diffusion ecological models well simulate the real ecosystem, and ([7-21]). Particularly, reaction-diffusion ecosystem were studied in [11-21]. For example, in [12], a single-species Markovian jumping ecosystem with diffusion and delayed feedback under Dirichlet boundary value was investigated:

$$
\left\{\begin{aligned}
\frac{\partial u(t, x)}{\partial t} & =q \Delta u(t, x)+u(t, x)[a-b u(t, x)]+c(r(t))[u(t, x)-u(t-\tau(t), x)], \quad t \geqslant 0, x \in \Omega \\
u(t, x) & =0, \quad x \in \partial \Omega, t \geqslant 0 .
\end{aligned}\right.
$$

Markov systems often occurred in various engineering technologies (see, e.g. [24-26]). Particularly, Markovian jumping delayed feedback model reflects well the influence of stochastic factors on time delays of the changes of populations, such as weather, temperature, humidity, ventilation status, and 
so on. But the case of Neumann boundary value on a single-species ecosystem is seldom researched. In fact, Neumann zero boundary value model well simulates the biosphere boundary without population migration. For example, freshwater fish do not enter the sea through rivers. Inspired by some ideas or methods of the related literature [11-28, 30], the author is to investigate the stability of a single-species Markovian jumping ecosystem with diffusion and delayed feedback under Neumann zero boundary value.

For convenience, throughout of this paper, $\Omega \subset R^{N}(1 \leqslant N \leqslant 3)$ is denoted as a bounded domain with a smooth boundary $\partial \Omega, \bar{\Omega}=\Omega \bigcup \partial \Omega$. Denote by $\|u\|_{H}=\sqrt{\int_{\Omega}|\nabla u(x)|^{2} d x}$ the norm of $H_{0}^{1}(\Omega)$, and by $\lambda_{1}$ the first positive eigenvalue of Laplace operator $-\Delta$ in $H_{0}^{1}(\Omega)$. Besides, we denote $|v|=\left(\left|v_{1}\right|,\left|v_{2}\right|\right)^{T}$ for $v=\left(v_{1}, v_{2}\right)^{T} \in \mathbb{R}^{2}$, and $|C|=\left(\left|c_{i j}\right|\right)_{2 \times 2}$ for matrix $C=\left(c_{i j}\right)_{2 \times 2}$. $\mathbb{N}$ represents the set of natural numbers $\{1,2, \cdots\}$. Denote by $L^{2}(\Omega)$ the space of all real-valued square integrable functions with the inner product $\langle\chi, \eta\rangle=\int_{\Omega} \chi(x) \eta(x) d x$, for $\chi, \eta \in L^{2}(\Omega)$ which derives the norm $\|\chi\|=\left(\int_{\Omega} \chi^{2}(x) d x\right)^{\frac{1}{2}}$ for $\chi \in L^{2}(\Omega)$. Denote by $\Delta=\sum_{j=1}^{m} \frac{\partial^{2}}{\partial x_{j}^{2}}$ the Laplace operator, with domain $\mathcal{D}(\Delta)=W_{0}^{1,2}(\Omega) \cap W_{0}^{2,2}(\Omega)$, which generates a strongly continuous semigroup $e^{t \Delta}$, where $W_{0}^{1,2}(\Omega)$ and $W_{0}^{2,2}(\Omega)$ are the Sobolev spaces with compactly supported sets. Next, the definition of the mild solution of the system (9) will be introduced. For convenience, the author takes $U=\{U(t, \cdot)\}_{[0, T]}$ for any given $T>0$ such that $U(t)$ is a $L^{2}(\Omega)$-valued function.

\section{System descriptions}

Denote by $(Y, \mathcal{F}, \mathbb{P})$ the complete probability space with a natural filtration $\left\{\mathcal{F}_{t}\right\}_{t \geq 0}$. Let $S=$ $\left\{1,2, \cdots, n_{0}\right\}$ and the random form process $\{r(t):[0,+\infty) \rightarrow S\}$ be a homogeneous, finite-state Markovian process with right continuous trajectories with generator $\Pi=\left(\gamma_{i j}\right)_{n_{0} \times n_{0}}$ and transition probability from mode $i$ at time $t$ to mode $j$ at time $t+\delta, i, j \in S$,

$$
\mathbb{P}(r(t+\delta)=j \mid r(t)=i)=\left\{\begin{array}{l}
\gamma_{i j} \delta+o(\delta), \quad j \neq i \\
1+\gamma_{i j} \delta+o(\delta), \quad j=i
\end{array}\right.
$$

where $\gamma_{i j} \geqslant 0$ is transition probability rate from $i$ to $j(j \neq i)$ and $\gamma_{i i}=-\sum_{j=1, j \neq i}^{n_{0}} \gamma_{i j}, \delta>0$ and $\lim _{\delta \rightarrow 0} o(\delta) / \delta=0$.

Consider the following ecosystem with diffusion and delayed feedback

$$
\left\{\begin{aligned}
\frac{\partial u(t, x)}{\partial t} & =q \Delta u(t, x)+u(t, x)[a-b u(t, x)]-c(r(t))[u(t, x)-u(t-\tau(t), x)], \quad t \geqslant 0, x \in \Omega \\
\frac{\partial u(t, x)}{\partial x} & =0, \quad x \in \partial \Omega, t \geqslant 0 \\
u(s, x) & =\Gamma(s, x), \quad(s, x) \in[-\tau, 0] \times \Omega
\end{aligned}\right.
$$

where $a>0$ and $b>0$ describe the growth rate and the intra-specific competition. Besides, the initial value function $\Gamma(s, x)$ is bounded on $[-\tau, 0] \times \Omega$. For convenience, $c(r(t))$ is denoted simply by $c_{r}$ for $r \in S$.

In addition, due to the limited resources of nature, the population density should have an upper limit. At the same time, the population density should also have a lower limit. For example, if the population density of whales is less than a certain degree, the population will become extinct, because male whales and female whales cannot meet in the sea. So the following assumption is suitable:

(H1) There exist two positive constants $N_{1}$ and $N_{2}$ with $N_{1} \leqslant \frac{a}{b} \leqslant N_{2}$ such that

$$
0<N_{1} \leqslant u(t, x) \leqslant N_{2}, \quad \forall x \in \bar{\Omega}, t \geqslant-\tau
$$

Definition 1. Set $u_{*}(t, x) \equiv u_{*}(x), \forall(t, x) \in[-\tau,+\infty) \times \bar{\Omega}$, then $u_{*}(x)$ is said to be a stationary solution of the ecosystem system (5) if $u_{*}(x)$ satisfies the boundedness assumption (H1), and 


$$
\left\{\begin{array}{l}
q \Delta u_{*}(x)+u_{*}(x)\left[a-b u_{*}(x)\right]=0, \quad t \geqslant 0, x \in \Omega, \\
\frac{\partial u_{*}(x)}{\partial x}=0, \quad x \in \partial \Omega, t \geqslant 0 .
\end{array}\right.
$$

Now, people can easily conclude from Definition 1 that $u_{*} \equiv \frac{a}{b}$ is a stationary solution of the ecosystem system (5). Moreover, setting $U(t, x)=u(t, x)-u_{*}$, then the system (5) is translated into

$$
\left\{\begin{aligned}
\frac{\partial U(t, x)}{\partial t}=q \Delta U(t, x)-a U(t, x)-b U^{2}(t, x)-c_{r}[U(t, x)-U(t-\tau(t), x)], \quad t \geqslant 0, x \in \Omega, \\
\frac{\partial U(t, x)}{\partial x}=0, \quad x \in \partial \Omega, t \geqslant 0, \\
U(s, x)=\Gamma(s, x)-\frac{a}{b}, \quad(s, x) \in[-\tau, 0] \times \Omega,
\end{aligned}\right.
$$

where the zero solution of the system (8) is corresponding to the positive stationary solution $u_{*} \equiv \frac{a}{b}$ of the ecosystem (5). And hence, the stability of the above-mentioned zero solution will be investigated below. Furthermore, applying an impulse control on the natural ecosystem (5) or (8) results in

$$
\left\{\begin{array}{l}
\frac{\partial U(t, x)}{\partial t}=q \Delta U(t, x)-a U(t, x)-b U^{2}(t, x)-c_{r}[U(t, x)-U(t-\tau(t), x)], \quad t \geqslant 0, t \neq t_{k}, x \in \Omega, \\
U\left(t_{k}^{+}, x\right)=M_{k} U\left(t_{k}^{-}, x\right), \quad k \in \mathbb{N}, \\
\frac{\partial U(t, x)}{\partial x}=0, \quad x \in \partial \Omega, t \geqslant 0, \\
U(s, x)=\xi(s, x)=\Gamma(s, x)-\frac{a}{b}, \quad(s, x) \in[-\tau, 0] \times \Omega,
\end{array}\right.
$$

whose zero solution is corresponding to the equilibrium point $u_{*} \equiv \frac{a}{b}$ of the following system:

$$
\left\{\begin{aligned}
\frac{\partial u(t, x)}{\partial t} & =q \Delta u(t, x)-a\left[u(t, x)-\frac{a}{b}\right]-b\left[u(t, x)-\frac{a}{b}\right]^{2}-c_{r}[u(t, x)-u(t-\tau(t), x)], \quad t \geqslant 0, t \neq t_{k}, x \in \Omega, \\
u\left(t_{k}^{+}, x\right)-\frac{a}{b} & =M_{k}\left[u\left(t_{k}^{-}, x\right)-\frac{a}{b}\right], \quad k \in \mathbb{N} \\
\frac{\partial u(t, x)}{\partial x} & =0, \quad x \in \partial \Omega, t \geqslant 0 \\
u(s, x) & =\Gamma(s, x), \quad(s, x) \in[-\tau, 0] \times \Omega
\end{aligned}\right.
$$

where each $t_{k}(k \in \mathbb{N})$ is a fixed impulsive instant with $0<t_{1}<t_{2}<\cdots, u\left(t_{k}^{+}, x\right)=\lim _{t \rightarrow t_{k}^{+}} u(t, x)$, and $u\left(t_{k}^{-}, x\right)=\lim _{t \rightarrow t_{k}^{-}} u(t, x)=u\left(t_{k}, x\right)$

Definition 2. For an arbitrarily given $T>0$, a $L^{2}(\Omega)$-valued function $U=\{U(t)\}_{[0, T]}$ is called a mild solution of (9) if $U(t, x) \in \mathcal{C}\left([0, T] ; L^{2}(\Omega)\right)$ satisfies $\int_{0}^{t}\|U(s)\|^{p} d s<\infty, i=1,2$, and the following integral equations hold for any $t \in[0, T]$ and $x \in \Omega$,

$$
\begin{aligned}
U(t, x)= & e^{q t \Delta} \xi(0, x)+\int_{0}^{t} e^{q(t-s) \Delta}\left(-a U(s, x)-b U^{2}(s, x)-c_{r}[U(s, x)-U(s-\tau(s), x)]\right) d s \\
& +e^{q t \Delta} \sum_{0<t_{k}<t} e^{-q t_{k} \Delta}\left(M_{k}-1\right) U\left(t_{k}, x\right), \quad t \geqslant 0,
\end{aligned}
$$

and

$$
\begin{gathered}
\frac{\partial U}{\partial v}=0, \quad x \in \partial \Omega, t \geqslant 0, \\
U(s, x)=\xi(s, x), \quad s \in[-\tau, 0], x \in \Omega .
\end{gathered}
$$

Remark 1. Definition 2 is well defined in view of [22] and [23].

In this paper, the following condition is also required: 
(H2) $\left\|e^{t \Delta}\right\|_{2} \leqslant M e^{-\gamma t}$, where both $M>0$ and $\gamma>0$ are constants, where $\left\|e^{t \Delta}\right\|_{2}=\sup _{\|w\|=1}\left\|e^{t \Delta} w\right\|$ (see [22]).

Lemma 1.(see, e.g. [30]). Let $\Omega$ be a bounded domain of $R^{m}$ with a smooth boundary $\partial \Omega$ of class $\mathcal{C}^{2}$ by $\Omega . v(x)$ is a real-valued function belonging to $H_{0}^{1}(\Omega)$ and $\left.\frac{\partial v(x)}{\partial v}\right|_{\partial \Omega}=0$. Then

$$
\lambda_{1} \int_{\Omega}|v(x)|^{2} d x \leqslant \int_{\Omega}|\nabla v(x)|^{2} d x
$$

which $\lambda_{1}$ is the smallest positive eigenvalue of the Neumann boundary problem

$$
\left\{\begin{array}{l}
-\Delta \varphi=\lambda \varphi, \quad x \in \Omega \\
\frac{\partial \varphi(x)}{\partial v}=0, \quad x \in \partial \Omega
\end{array}\right.
$$

where $v$ represents the external normal direction of $\partial \Omega$.

Lemma 2 (Banach contraction mapping principle [29]) Let $\Theta$ be a contraction operator on a complete metric space $\amalg$, then there exists a unique point $u \in \amalg$ for which $\Theta(u)=u$.

\section{Main result}

Firstly, the unique existence of the stationary solution of the ecosystem (5) should be considered. Moreover, the unique stationary solution of the ecosystem should be positive. Based on the two point, the author presents the following unique existence theorem:

Theorem 1. Suppose (H1) holds. For all $r \in S$, the system (5) possesses a positive stationary solution $u_{*} \equiv \frac{a}{b}$ for all $(t, x) \in[-\tau,+\infty) \times \bar{\Omega}$. If, in addition, the following condition is satisfied:

$$
a<\lambda_{1} q+2 b N_{1}
$$

then the positive solution $u_{*}$ is the unique stationary solution of the system (5).

Proof. Obviously, for $(t, x) \in[-\tau, 0] \times \bar{\Omega}, u_{*} \equiv \frac{a}{b}$ makes the following equations hold:

$$
q \Delta u_{*}+u_{*}\left[a-b u_{*}\right]=0, \quad t \geqslant 0, x \in \Omega,
$$

and

$$
\frac{\partial u_{*}}{\partial x}=0, \quad x \in \partial \Omega, t \geqslant 0 .
$$

Thus, Definition 1 yields that $u_{*}>0$ defined in Theorem 1 is the unique stationary solution of the system (5).

Below, the author claims that $u_{*}$ is the unique stationary solution of the ecosystem (5).

Indeed, if $u_{*}$ and $v_{*}(x)$ are two different stationary solutions of the system (5), then Poincare inequality and the boundary condition yield

$$
a \int_{\Omega}\left(u_{*}-v_{*}(x)\right)^{2} d x-b \int_{\Omega}\left(u_{*}-v_{*}(x)\right)^{2}\left(u_{*}+v_{*}(x)\right) d x \geqslant \lambda_{1} q \int_{\Omega}\left|u_{*}-v_{*}(x)\right|^{2} d x
$$

The condition (12), Definition 2 and the continuity of $u_{*}$ and $v_{*}$ lead to

$$
a \int_{\Omega}\left(u_{*}-v_{*}(x)\right)^{2} d x-b \int_{\Omega}\left(u_{*}-v_{*}(x)\right)^{2}\left(u_{*}+v_{*}(x)\right) d x<\lambda_{1} \int_{\Omega}\left|u_{*}-v_{*}(x)\right|^{2} d x,
$$

which contradicts the inequality (13).

This completes the proof. 
Remark 2. As far as I am concerned, Theorem 1 is the first theorem to give the unique existence of the equilibrium point of a single-species ecosystem under Neumann boundary value.

Next, the global stability of the stationary solution $u_{*} \equiv \frac{a}{b}$ should be investigated.

Theorem 2. Set $p \geqslant 1$. Suppose all the conditions of Theorem 1 hold. Assume, in addition, the condition (H2) holds, and

$$
0<\omega<1
$$

then zero solution of the system (9) is globally exponential stability in the $p$ th moment, equivalently, $u_{*} \equiv \frac{a}{b}$ of the system (10) is globally exponential stability in the $p$ th moment. where $N_{0}=\max \left\{\mid N_{1}-\right.$ $\left.\frac{a}{b} \mid, N_{2}-\frac{a}{b}\right\}, \mu=\inf _{k \in \mathbb{N}}\left(t_{k+1}-t_{k}\right)>0$,

$$
\boldsymbol{\omega}=\left[4^{p-1}\left(\left(a+c_{r}\right)\left(\frac{M}{q \gamma}\right)^{p}+b\left(\frac{2 M N_{0}}{q \gamma}\right)^{p}+c_{r}\left(\frac{M}{q \gamma}\right)^{p}+M^{2 p}\left(\max _{k}\left|M_{k}-1\right|\right)^{p}\left(1+\frac{1}{q \gamma \mu}\right)^{p}\right)\right]^{\frac{1}{p}} .
$$

Proof. Banach contraction mapping principle will play a important role in this proof, so the author formulates a contraction mapping on a suitable complete metric space firstly.

Let $\amalg$ be the normed space consisting of functions $g(t, x):[-\tau,+\infty) \times \bar{\Omega} \rightarrow\left[N_{1}-\frac{a}{b}, N_{2}-\frac{a}{b}\right]$, where $g$ satisfies:

(A1) $g$ is $p$ th moment continuous at $t \geqslant 0$ with $t \neq t_{k}(k \in \mathbb{N})$;

(A2) for any given $x \in \Omega, \lim _{t \rightarrow t_{k}^{-}} g(t, x)$ and $\lim _{t \rightarrow t_{k}^{+}} g(t, x)$ exist, and $\lim _{t \rightarrow t_{k}^{-}} g(t, x)=g\left(t_{k}, x\right)$;

(A3) $g(s, x)=\xi(s, x), \quad \forall s \in[-\tau, 0], x \in \Omega$;

(A4) $e^{\alpha t}\|g(t)\|^{p} \rightarrow 0$ as $t \rightarrow+\infty$, where $\alpha$ is a positive scalar with $0<\alpha<q \gamma$.

It is not difficult to verify that the normed space $\amalg$ is a complete metric space if it is equipped with the following metric:

$$
\operatorname{dist}(U, V)=\left(\sup _{t \geqslant-\tau}\|U(t, x)-V(t, x)\|^{p}\right)^{\frac{1}{p}}, \quad \forall U, V \in \coprod
$$

Construct an operator $\Theta$ such that for any given $U \in \amalg$,

$$
\left\{\begin{aligned}
\Theta(U)(t, x)= & e^{q t \Delta} \xi(0, x)+\int_{0}^{t} e^{q(t-s) \Delta}\left(-a U(s, x)-b U^{2}(s, x)-c_{r}[U(s, x)-U(s-\tau(s), x)]\right) d s \\
& +e^{q t \Delta} \sum_{0<t_{k}<t} e^{-q t_{k} \Delta}\left(M_{k}-1\right) U\left(t_{k}, x\right), \quad t \geqslant 0, \\
\frac{\partial \Theta(U)}{\partial v}=0, \quad x \in \partial \Omega, t \geqslant 0, & \\
\Theta(U)(s, x)= & \xi(s, x), \quad s \in[-\tau, 0], x \in \Omega .
\end{aligned}\right.
$$

Below, it is necessary to show that $\Theta: \amalg \rightarrow \amalg$, which may require four steps to achieve the goal.

Step 1. The author claim that for $U \in \amalg, \Theta(U)$ must be $p$ th moment continuous at $t \geqslant 0$ with $t \neq t_{k}(k \in \mathbb{N})$.

Indeed, $U \in\left[N_{1}-\frac{a}{b}, N_{2}-\frac{a}{b}\right]$ means the boundedness of $U$, and let $\delta$ be small enough scalar, a routine proof yields that if $\delta \rightarrow 0$, for $t \in[0,+\infty) \backslash\left\{t_{k}\right\}_{k=1}^{\infty}$,

$$
\begin{aligned}
& \|\Theta(U)(t+\delta, x)-\Theta(U)(t, x)\|^{p} \leqslant 4^{p-1}\left\|e^{q(t+\delta) \Delta} \xi(0, x)-e^{q t \Delta} \xi(0, x)\right\|^{p} \\
& +4^{p-1}\left\|\int_{0}^{t+\delta} e^{q(t+\delta-s) \Delta}\left[-a U(s, x)-b U^{2}(s, x)\right] d s-\int_{0}^{t} e^{q(t-s) \Delta}\left[-a U(s, x)-b U^{2}(s, x)\right] d s\right\|^{p} \\
& +4^{p-1}\left\|\int_{0}^{t+\delta} e^{q(t+\delta-s) \Delta}\left[c_{r}(U(s, x)-U(s-\tau(s), x))\right] d s-\int_{0}^{t} e^{q(t-s) \Delta}\left[c_{r}(U(s, x)-U(s-\tau(s), x))\right] d s\right\|^{p} \\
& +4^{p-1}\left\|e^{q(t+\delta) \Delta} \sum_{0<t_{k}<t+\delta} e^{-q t_{k} \Delta}\left(M_{k}-1\right) U\left(t_{k}, x\right)-e^{q t \Delta} \sum_{0<t_{k}<t} e^{-q t_{k} \Delta}\left(M_{k}-1\right) U\left(t_{k}, x\right)\right\|^{p} \rightarrow 0,
\end{aligned}
$$


which proves the claim. And then (A1) is verified.

Step 2. Verifying $\Theta(U)$ satisfies (A2), where $U \in \amalg$.

In fact, for $U \in \amalg$, people can easily see it from (17) that $\lim _{t \rightarrow t_{k}^{-}} \Theta(U)(t, x)$ and $\lim _{t \rightarrow t_{k}^{+}} \Theta(U)(t, x)$ exist, and $\lim _{t \rightarrow t_{k}^{-}} \Theta(U)(t, x)=\Theta(U)\left(t_{k}, x\right)$, which verifies (A2).

Step 3. Verifying $\Theta(U)$ satisfies (A3), where $U \in \amalg$. Indeed, the third equation of (17) verifies (A3) directly.

Step 4. Verifying (A4), i.e., for $U \in \amalg$, verifying

$$
e^{\alpha t}\|\Theta(U)(t)\|^{p} \rightarrow 0, \text { if } t \rightarrow+\infty .
$$

Indeed,

$$
\begin{aligned}
& e^{\alpha t}\|\Theta(U)(t, x)\|^{p}=e^{\alpha t} \| e^{q t \Delta} \xi(0, x)+\int_{0}^{t} e^{q(t-s) \Delta}\left(-a U(s, x)-b U^{2}(s, x)-c_{r}[U(s, x)-U(s-\tau(s), x)]\right) d s \\
& +e^{q t \Delta} \sum_{0<t_{k}<t} e^{-q t_{k} \Delta}\left(M_{k}-1\right) U\left(t_{k}, x\right) \|^{p} \\
& \leqslant 5^{p-1} e^{\alpha t}\left\|e^{q t \Delta} \xi(0, x)\right\|^{p}+5^{p-1} e^{\alpha t}\left\|\int_{0}^{t} e^{q(t-s) \Delta}\left[-a U(s, x)-b U^{2}(s, x)\right] d s\right\|^{p}+5^{p-1} e^{\alpha t}\left\|\int_{0}^{t} e^{q(t-s) \Delta} c_{r} U(s, x) d s\right\|^{p} \\
& +5^{p-1} e^{\alpha t}\left\|\int_{0}^{t} e^{q(t-s) \Delta} c_{r} U(s-\tau(s), x) d s\right\|^{p}+5^{p-1} e^{\alpha t}\left\|e^{q t \Delta} \sum_{0<t_{k}<t} e^{-q t_{k} \Delta}\left(M_{k}-1\right) U\left(t_{k}, x\right)\right\|^{p}, t \geqslant 0,
\end{aligned}
$$

Moreover,

$$
e^{\alpha t}\left\|e^{q t \Delta} \xi(0, x)\right\|^{p} \leqslant M^{p} e^{\alpha t} e^{-\gamma q t}\|\xi(0, x)\|^{p} \rightarrow 0, \text { if } t \rightarrow+\infty .
$$

$U \in \amalg$ means $U \in\left[N_{1}-\frac{a}{b}, N_{2}-\frac{a}{b}\right]$, and

$$
U^{2} \leqslant N_{0}|U|, \quad \text { where } N_{0}=\max \left\{\left|N_{1}-\frac{a}{b}\right|, N_{2}-\frac{a}{b}\right\}
$$

and Holder inequality yield

$$
\begin{aligned}
& e^{\alpha t}\left\|\int_{0}^{t} e^{q(t-s) \Delta}\left(-a U(s, x)-b U^{2}(s, x)\right) d s\right\|^{p} \\
\leqslant & 2^{p-1} M^{p}\left[a^{p}\left(\frac{1}{q \gamma}\right)^{p-1} \int_{0}^{t} e^{-(q \gamma-\alpha)(t-s)} e^{\alpha s}\|U(s, x)\|^{p} d s+b^{p} N_{0}^{p}\left(\frac{1}{q \gamma}\right)^{p-1} \int_{0}^{t} e^{-q \gamma(t-s)}\|U\|^{p} d s\right] .
\end{aligned}
$$

On the other hard, $e^{\alpha t}\left\|U_{i}(t)\right\|^{p} \rightarrow 0$ means that for any $\varepsilon>0$, there exists $t^{*}>0$ such that all $e^{\alpha t}\left\|U_{i}(t)\right\|^{p}<\varepsilon$. And so,

$$
\begin{aligned}
& \int_{0}^{t} e^{-(q \gamma-\alpha)(t-s)} e^{\alpha s}\|U(s, x)\|^{p} d s \\
\leqslant & \max _{s \in\left[0, t^{*}\right]}\left(e^{\alpha s}\|U(s, x)\|^{p}\right) e^{-(q \gamma-\alpha) t} \frac{1}{q \gamma-\alpha} e^{(q \gamma-\alpha) t^{*}}+\varepsilon \frac{1}{q \gamma-\alpha},
\end{aligned}
$$

which together with the arbitrariness of $\varepsilon$ implies that

$$
\int_{0}^{t} e^{-(q \gamma-\alpha)(t-s)} e^{\alpha s}\|U(s, x)\|^{p} d s \rightarrow 0, \quad t \rightarrow+\infty
$$


Similarly as the proof of (23), people can prove

$$
\begin{gathered}
e^{\alpha t}\left\|\int_{0}^{t} e^{q(t-s) \Delta}\left[-a U(s, x)-b U^{2}(s, x)\right] d s\right\|^{p}, \quad t \rightarrow+\infty . \\
e^{\alpha t}\left\|\int_{0}^{t} e^{q(t-s) \Delta} c_{r} U(s, x) d s\right\|^{p}, \quad t \rightarrow+\infty
\end{gathered}
$$

Due to $U(s, x)=\xi(s, x)$ is bounded on $[-\tau, 0] \times \Omega$, it is not difficult to prove similarly

$$
e^{\alpha t}\left\|\int_{0}^{t} e^{q(t-s) \Delta} c_{r} U(s-\tau(s), x) d s\right\|^{p}, \quad t \rightarrow+\infty .
$$

Next, using the definition of Riemann integral $\int_{a}^{b} e^{s} d s$ results in

$$
\begin{aligned}
& e^{\alpha t}\left\|e^{q t \Delta} \sum_{0<t_{k}<t} e^{-q t_{k} \Delta}\left(M_{k}-1\right) U\left(t_{k}, x\right)\right\|^{p} \\
\leqslant & 2^{p-1} \max _{k}\left|M_{k}-1\right|\left[e^{-(p q \gamma-\alpha) t}\left(\sum_{0<t_{k} \leqslant t^{*}} e^{q \gamma t_{k}}\left\|U\left(t_{k}, x\right)\right\|\right)^{p}+\varepsilon \frac{1}{\left(q \gamma-\frac{\alpha}{p}\right)^{p}}\right] \rightarrow 0 .
\end{aligned}
$$

Combining (20)-(28) yields (19).

It follows from the above four steps that

$$
\Theta(\amalg) \subset \amalg .
$$

Finally, the author claims that $\Theta$ is a contractive mapping on $\amalg$. Indeed, for any $U, V \in \amalg$, Holder inequality and $(\mathrm{H} 2)$ yield

$$
\begin{aligned}
& \left\|\int_{0}^{t} e^{q(t-s) \Delta}[U(s, x)-V(s, x)] d s\right\|^{p} \\
\leqslant & \left(M \int_{0}^{t} e^{-q \gamma(t-s)}\|U(s, x)-V(s, x)\| d s\right)^{p} \\
\leqslant & M^{p}\left(\left(\frac{1}{q \gamma}\right)^{\frac{p-1}{p}}\left(\frac{1}{q \gamma}\right)^{\frac{1}{p}}\left[\sup _{t \geqslant-\tau}\|U(t, x)-V(t, x)\|^{p}\right]^{\frac{1}{p}}\right)^{p} \\
\leqslant & \left(\frac{M}{q \gamma}\right)^{p}[\operatorname{dist}(U, V)]^{p} .
\end{aligned}
$$

Similarly,

$$
\begin{aligned}
& \left\|\int_{0}^{t} e^{q(t-s) \Delta}\left[U^{2}(s, x)-V^{2}(s, x)\right] d s\right\|^{p} \\
\leqslant & \left(2 M N_{0} \int_{0}^{t} e^{-q \gamma(t-s)}\|U(s, x)-V(s, x)\| d s\right)^{p} \\
\leqslant & \left(\frac{2 M N_{0}}{q \gamma}\right)^{p}[\operatorname{dist}(U, V)]^{p},
\end{aligned}
$$

and

$$
\left\|\int_{0}^{t} e^{q(t-s) \Delta}[U(s-\tau(s), x)-V(s-\tau(s), x)] d s\right\|^{p} \leqslant\left(\frac{M}{q \gamma}\right)^{p}[\operatorname{dist}(U, V)]^{p}
$$


Suppose $t_{j-1}<t \leqslant t_{j}$, then the definition of Riemann integral $\int_{a}^{b} e^{s} d s$ yields

$$
\begin{aligned}
& \left\|e^{q t \Delta} \sum_{0<t_{k}<t} e^{-q t_{k} \Delta}\left(M_{k}-1\right)\left[U\left(t_{k}, x\right)-V\left(t_{k}, x\right)\right]\right\|^{p} \\
\leqslant & M^{2 p}\left(\max _{k}\left|M_{k}-1\right|\right)^{p}\left[e^{-q \gamma t}\left(e^{q \gamma t_{j-1}}+\frac{1}{\mu} \sum_{0<t_{k} \leqslant t_{j-2}} e^{q \gamma t_{k}}\left(t_{k+1}-t_{k}\right)\right) \cdot \operatorname{dist}(U, V)\right]^{p} \\
\leqslant & M^{2 p}\left(\max _{k}\left|M_{k}-1\right|\right)^{p}\left(1+\frac{1}{q \gamma \mu}\right)^{p} \cdot[\operatorname{dist}(U, V)]^{p} .
\end{aligned}
$$

It follows from (30)-(33) that

$$
\begin{aligned}
& \|\Theta(U)-\Theta(V)\|^{p} \\
\leqslant & 4^{p-1}\left(a+c_{r}\right)\left\|\int_{0}^{t} e^{q(t-s) \Delta}[U(s, x)-V(s, x)] d s\right\|^{p}+4^{p-1} b\left\|\int_{0}^{t} e^{q(t-s) \Delta}\left[U^{2}(s, x)-V^{2}(s, x)\right] d s\right\|^{p} \\
& +4^{p-1} c_{r}\left\|\int_{0}^{t} e^{q(t-s) \Delta}[U(s-\tau(s), x)-V(s-\tau(s), x)] d s\right\|^{p} \\
& +4^{p-1}\left\|\int_{0}^{t} e^{q(t-s) \Delta} e^{q t \Delta} \sum_{0<t_{k}<t} e^{-q t_{k} \Delta}\left(M_{k}-1\right)\left[U\left(t_{k}, x\right)-V\left(t_{k}, x\right)\right]\right\|^{p} \\
\leqslant & 4^{p-1}\left(\left(a+c_{r}\right)\left(\frac{M}{q \gamma}\right)^{p}+b\left(\frac{2 M N_{0}}{q \gamma}\right)^{p}+c_{r}\left(\frac{M}{q \gamma}\right)^{p}+M^{2 p}\left(\max _{k}\left|M_{k}-1\right|\right)^{p}\left(1+\frac{1}{q \gamma \mu}\right)^{p}\right)[\operatorname{dist}(U, V)]^{p},
\end{aligned}
$$

which derives

$$
\operatorname{dist}(\Theta(U), \Theta(V)) \leqslant\left(\max _{r \in S} \omega_{r}\right) \operatorname{dist}(U, V), \quad \forall U, V \in \coprod,
$$

where $\omega_{r}$ satisfies $0<\omega_{r}<1$. This shows that $\Theta: \amalg \rightarrow \amalg$ is a contraction mapping such that there exists the fixed point $U$ of $\Theta$ in $\amalg$, which implies that $U$ is the solution of the system (9), satisfying $e^{\alpha t}\|U\|^{p} \rightarrow 0, t \rightarrow+\infty$ so that $e^{\alpha t}\left\|u-u_{*}\right\|^{p} \rightarrow 0, t \rightarrow+\infty$. Therefore, zero solution of the system (9) is globally exponential stability in the $p$ th moment, equivalently, $u_{*} \equiv \frac{a}{b}$ of the system (10) is globally exponential stability in the $p$ th moment.

Remark 3. As far as I am concerned, it is the first paper to apply the Laplacian semigroup theory to deal with the stability of a single-species ecosystem with Markovian jumping and delayed feedback.

\section{Numerical example}

Example 1. Set $S=\{1,2\}, c 1=0.1, c 2=0.06, q=0.2, a=0.5, b=0.2, N_{1}=2, N_{2}=3$, then $N_{0}=0.5, u_{*}=2.5$. Suppose, in addition, $\Omega=(0, \pi)$, then by computing the eigenfunctions of $-\Delta$, one can get $\left\|e^{t \Delta}\right\| \leqslant e^{-\pi^{2} t}, t \geqslant 0$, and so $\gamma=\pi^{2}=\lambda_{1}, M=1$. Direct computation yields

$$
0.5=a<2.7739=\lambda_{1} q+2 b N_{1},
$$

which implies that the condition (12) is satisfied. Theorem 1 yields that the positive solution $u_{*} \equiv 2.5$ is the unique stationary solution of the system (5).

Example 2.Suppose all the data of Example are applied to this example. Assume, in addition, $p=$ $1.5, M_{k} \equiv 1.02, \mu=5$, Obviously the condition (H2) holds in Example 1, and direct calculations yield:

$$
\omega_{1}=\left[4^{p-1}\left(\left(a+c_{1}\right)\left(\frac{M}{q \gamma}\right)^{p}+b\left(\frac{2 M N_{0}}{q \gamma}\right)^{p}+c_{1}\left(\frac{M}{q \gamma}\right)^{p}+M^{2 p}\left(\max _{k}\left|M_{k}-1\right|\right)^{p}\left(1+\frac{1}{q \gamma \mu}\right)^{p}\right)\right]^{\frac{1}{p}},
$$

and

$$
\omega_{2}=\left[4^{p-1}\left(\left(a+c_{2}\right)\left(\frac{M}{q \gamma}\right)^{p}+b\left(\frac{2 M N_{0}}{q \gamma}\right)^{p}+c_{2}\left(\frac{M}{q \gamma}\right)^{p}+M^{2 p}\left(\max _{k}\left|M_{k}-1\right|\right)^{p}\left(1+\frac{1}{q \gamma \mu}\right)^{p}\right)\right]^{\frac{1}{p}} .
$$


And hence the condition (14) is satisfied.

Thereby, Theorem 2 yields that zero solution of the system (9) is globally exponential stability in the $p$ th moment, equivalently, $u_{*} \equiv 2.5$ of the system (10) is globally exponential stability in the $p$ th moment.

\section{Conclusions and further considerations}

In this paper, there are some improvements on mathematical methods, for it is the first paper to employ fixed point theory, Laplacian semigroup theory and variational methods to solve the unique existence of the globally stable positive equilibrium point of a single-species ecosystem with Markovian jumping and delayed feedback. Numerical examples are given to show the feasibility of artificial management of nature by way of impulse control.

As pointed out in [28, Definition 1], the author originally proposed a class of global asymptotical stability in the meaning of switching in [27, Definition 3]. Particularly, in the case of one subsystem, the switched system becomes non-switched common system, the global asymptotical stability in the meaning of switching becomes that in the classical meaning. The author actually gave both of the mentioned two classes of global stability for the unique (positive) stationary solution in [27]. Now the author wants to know whether the two classes of global stability can be applied to ecosystem, and what meanings about the stability in the meaning of switching for an ecosystem ? This is an interesting problem. Besides, in [27, Statement 2], the author originally design an example to show that under the influence of diffusion, the unique equilibrium point of ordinary differential system with Lipschitz continuous activation function becomes at least three equilibrium points of its corresponding partial differential system. Now the author wants to know whether the unique equilibrium point is globally stable. Furthermore, the author in [27, Section 5] originally proposed four problems, particularly [27, Problem 1 ] and [27, Problem 4 ] can also be suitable for the case of Neumann boundary value in this paper. Such problems are also interesting. Moreover, how to consider the case of infinite delays in [10, Problem 6] to ecosystem ? It is also an interesting problem. To sum up, the following problems are more interesting:

Problem 1. Is the zero solution of the ordinary differential equation in [21, Theorem 3] or [27, Statement 2] global stable?

Problem 2. How to design another example somewhat similar to [21, Theorem 3] or [27, Statement 2] with Lipschitz continuous activation function, where the ordinary differential equation possesses a globally stable equilibrium point, but its corresponding partial differential equation owns at least two stationary solutions.

Acknowledgements Thank Chinese science and Technology Department of Sichuan Province for funding this work through the Application basic research project (No. 2020YJ0434), and thank Chengdu Normal University for funding this work through Major scientific research projects of Chengdu Normal University (No. CS19ZDZ01).

Conflicts of Interest: The author declares no conflict of interest.

\section{References}

1. L. Chen, X. Meng, and J. Jiao, Biodynamics, Science Press, Beijing, China, 2009.

2. X. Zou and K. Wang, "A robustness analysis of biological population models with protection zone," Applied Mathematical Modelling, vol.35, pp. 5553-5563, 2011.

3. W. Ji and G. Hu, "Stability and explicit stationary density of a stochastic single-species model," Applied Mathematics and Computation, vol.390, 125593, 2021.

4. X. Yu , S. Yuan, and T. Zhang, "Persistence and ergodicity of a stochastic single species model with allee effect under regime switching, " Communications in Nonlinear Science and Numerical Simulation, vol.59, pp. 359-374, 2018. 
5. Y. Jin , " Analysis of a stochastic single species model with allee effect and jump-diffusion," Advances in Difference Equations, vol.165, pp.1-11, 2020.

6. G.F. Gause, The Struggle for Existence, Williams and Wilkins, Baltimore, MD, 1935.

7. J. Huisman, M. Arrayas, U. Ebert, and B. Sommeijer, "How do sinking phytoplankton species manage to persist?" The American Naturalist, vol. 159, pp. 245-254, 2002.

8. R.H. MacArthur, and E.O. Wilson, The Theory of Island Biogeography, Princeton University Press, Princeton, NJ, 1967.

9. J.G. Skellam, "Random dispersal in theoretical populations," Biometrika, vol.38, pp.196-218, 1951.

10. R. Rao, J. Huang, X. Li. Stability analysis of nontrivial stationary solution of reaction-diffusion neural networks with time delays under Dirichlet zero boundary value. Neurocomputing, 445C (2021) 105-120.

11. Youshan Tao and Michael Winkler. Boundedness and stabilization in a population model with cross-diffusion for one species, Proc. London Math. Soc. (3) 119 (2019) 1598-1632.

12. Ruofeng Rao, Existence, Uniqueness, and Input-to-State Stability of Ground State Stationary Strong Solution of a Single-Species Model via Mountain Pass Lemma, Complexity, 2021

13. Chakraborty, K and Manthena, V. Modelling and analysis of spatio-temporal dynamics of a marine ecosystem. NONLINEAR DYNAMICS, 2015, 81 (4) , pp.1895-1906

14. Kabir, MH. Reaction-diffusion modeling of the spread of spruce budworm in boreal ecosystem. JOURNAL OF APPLIED MATHEMATICS AND COMPUTING, 2021, 66 (1-2) , pp.203-219

15. Robbin Bastiaansen, Olfa Jalbi, Vincent Deblauwe, Maarten B. Eppinga, Koen Siteur, Eric Siero, Stephane Mermoz, Alexandre Bouvet, Arjen Doelman, and Max Rietkerk. Multistability of model and real dryland ecosystems through spatial self-organization,PROCEEDINGS OF THE NATIONAL ACADEMY OF SCIENCES OF THE UNITED STATES OF AMERICA, 2018, Volume115 Issue44 Page11256-11261

16. R. Rao, X. Yang, R. Tang, Y. Zhang, X. Li. Impulsive stabilization and stability analysis for Gilpin-Ayala competition model involved in harmful species via LMI approach and variational methods. Mathematics and Computers in Simulation, 188C (2021) 571-590.

17. R. Rao, Q. Zhu and K. Shi, Input-to-State Stability for Impulsive Gilpin-Ayala Competition Model With Reaction Diffusion and Delayed Feedback, IEEE Access, 2020, 8, 222625-222634.

18. Youshan Tao and Michael Winkler. Boundedness and stabilization in a population model with cross-diffusion for one species, Proc. London Math. Soc. (3) 119 (2019) 1598-1632.

19. Xiang, H., Liu, B., Fang, Z . Optimal control strategies for a new ecosystem governed by reaction-diffusion equations. Journal of Mathematical Analysis And Applications, 2018, Volume467,Issue1,Page270-291

20. R. Rao. Mathematical Methods in the Applied Sciences Impulsive control and global stabilization of reaction-diffusion epidemic model. Mathematical Methods in the Applied Sciences, 2021, DOI. 10.1002/mma.7517

21. Rao, R. Global Stability of Delayed Ecosystem via Impulsive Differential Inequality and Minimax Principle. Mathematics, 2021, 9, 1943.

22. Winkler M. Aggregation vs. global diffusive behavior in the higher-dimensional Keller-Segel model. J Diff Equ. 2010; 248: 2889-2905.

23. R. Rao, S. Zhong, P. Pu. LMI-based robust exponential stability criterion of impulsive integro-differential equations with uncertain parameters via contraction mapping theory. Adv Diff Equ. 2017; 2017:19.

24. Lizbeth Naranjo,Luz Judith R. Esparza and Carlos J. Perez. A Hidden Markov Model to Address Measurement Errors in Ordinal Response Scale and Non-Decreasing Process. Mathematics 2020, 8(4), 622; https:/ / doi.org/10.3390/math8040622 - 17 Apr 2020

25. Andrey Borisov andIgor Sokolov. Optimal Filtering of Markov Jump Processes Given Observations with State-Dependent Noises: Exact Solution and Stable Numerical Schemes. Mathematics 2020, 8(4), 506; https:/ / doi.org/10.3390/math8040506

26. Pierre Hodara and Ioannis Papageorgiou. Poincare-Type Inequalities for Compact Degenerate Pure Jump Markov Processes. Mathematics 2019, 7(6), 518; https:/ / doi.org/10.3390/math7060518 - 06 Jun 2019

27. R. Rao. Stability Analysis of Nontrivial Stationary Solution and Constant Equilibrium Point of Reaction-Diffusion Neural Networks with Time Delays under Dirichlet Zero Boundary Value. Preprints, 2020, 2020040277 (doi: 10.20944/preprints202004.0277.v6).

28. R. Rao, X. Li. Input-to-state stability in the meaning of switching for delayed feedback switched stochastic financial system. AIMS Mathematics, 6(1): 1040-1064. 
29. Istratescu VI. Fixed point theory, in: an introduction. D.Reidel, the Netherlands, 1981 .

30. Pan J, Liu XZ, Zhong SM. Stability criteria for impulsive reaction-diffusion Cohen-Grossberg neural networks with time-varying delays. Math Comput Model. 2010; 51: 1037-1050. 